\title{
IMPACT OF MOTOR IMAGERY AT SPINAL LEVEL
}

\author{
Grospretre, S., Lebon, F., Papaxanthis, C. and Martin, A. \\ INSERM U1093
}

Introduction: Motor imagery (MI), the mental representation of actual contraction, was shown to activate same cortical structures as actual contraction without producing movement (Lotze et al. 1999). However, the question of MI effect at a spinal level is not still resolved, since results found with an H-reflex technique vary within the literature. It was previously shown that a sub-threshold cortical output could modulate spinal excitability without activating alpha-motoneurons (Grospretre et al. 2014). Because spinal interneurons have excitability threshold lower than motoneurons (Daniele \& McDermott 2009), it could be hypotheses that MI induces a cortical output that is too low to activate motoneuronal pool but sufficient to activate spinal interneurons. Thus, the aim of this study was to evaluate the impact of imagined eccentric, concentric and isometric contraction on presynaptic inhibition. This study could also show if eccentric, concentric or isometric contraction share same cortico-spinal pathways.

Method: 8 healthy young subjects participated in this study. H-reflexes and Mwaves of soleus muscle (SOL) were elicited by electrical stimulations of the posterior tibial nerve. SOL H-reflexes were conditioned by stimulation of the common peroneal nerve in order to induce D1 presynatic inhibition of SOL Ia afferences onto alpha motoneurons. Conditioning-test interval used was $21 \mathrm{~ms}$ (Achache et al. 2010). Conditioned and unconditioned H-reflexes were elicited during rest and during MI of eccentric, concentric and isometric contractions of the SOL muscle.

Results: Whatever the imagined contraction type, no effect of MI was found on SOL EMG activity as well as on H test reflexes. However, conditioned H-reflexes statistically increased between rest and imagined contraction. At rest, conditioned H-reflexes were $16 \%$ lower than unconditioned $(\mathrm{p}<0.05)$. During motor imagery, whatever the imagined contraction type, no differences were found between conditioned and unconditioned H-reflexes.

Discussion: The lack of EMG activity or H-reflex modulation showed that alpha-motoneurons were not activated during MI. However, a significant impact on presynaptic inhibition of SOL Ia afferences was found for all imagined conditions. This result demonstrates that $\mathrm{MI}$ of muscle contraction induces the activation of corticospinal pathway that is too low to activate motoneuron pool but sufficient to activate primary afferent depolarization interneurons mediating presynaptic inhibition.

\section{References:}

Achache et al. (2010), Brain, 133: 1470-1483

Daniele \& McDermott (2009), J Neuroscience, 29(3): 686-695

Grospretre et al. (2014), Neuroscience, 263: 60-71

Lotze et al. (1999), J Cogn Neurosci, 11: 491-501

Contact sidney.grospretre@gmail.com 